\title{
The Application of Hand-Held Ultrasound Scanner in Teaching of Telemedicine and Rural Medicine
}

1Justin Wright, ${ }^{2}$ Oscar Noriega, ${ }^{3} \mathrm{Hoi}$ Ho

\begin{abstract}
Stethoscope, the symbol of modern medicine has been in place for more than 150 years. However, this reliable diagnostic instrument has started showing its age with the arrival of a newer diagnostic instrument: the hand-held ultrasound.

Hand-held ultrasound is rapidly expanding in many specialties but not yet in obstetrics and gynecology. There is a paucity of literature on the application of hand-held ultrasound in obstetrics and gynecology, in contrast to a large volume of literature published by other specialties. Several studies demonstrated that medical students and residents can improve the accuracy of their physical examinations with the use of hand-held ultrasound. Similarly, physical examinations performed by hospitalists with hand-carried ultrasound are more accurate than those performed without the instrument. Advances in technology have contributed to the improvement of image quality and portability. As a result, these devices become increasingly available in many clinical settings such as point of care, telemedicine and rural medicine. The quality of ultrasound images of the hand-held scanner is inferior to those of standard laptop ultrasound. However, the hand-held ultrasound requires much less time for setup.

Although there have been recommendations for training guidelines for operators of hand-held ultrasound, standards for optimal training to achieve level of competency have not been determined.
\end{abstract}

Keywords: Hand-held ultrasound, Hand-carried ultrasound, Telemedicine, Rural medicine, Quality and accuracy of handheld ultrasound, Physical examination, Clinical competence.

How to cite this article: Wright $\mathrm{J}$, Noriega $\mathrm{O}, \mathrm{Ho} \mathrm{H}$. The Application of Hand-Held Ultrasound Scanner in Teaching of Telemedicine and Rural Medicine. Donald School J Ultrasound Obstet Gynecol 2014;8(1):87-91.

Source of support: Nil

Conflict of interest: None

${ }^{1}$ Assistant Professor, ${ }^{2}$ Associate Professor, ${ }^{3}$ Professor

1,2Department of Family and Community Medicine, Paul L Foster School of Medicine, Texas Tech University Health Sciences Center at EI Paso, Texas, USA

${ }^{3}$ Department of Internal Medicine, Associate Dean for Faculty Affairs and Development, Director, Center for Advanced Teaching and Assessment in Clinical Simulation Paul L Foster School of Medicine, Texas Tech University Health Sciences Center at El Paso, Texas, USA

Corresponding Author: Justin Wright, Assistant Professor Department of Family and Community Medicine, Paul L Foster School of Medicine, Texas Tech University Health Sciences Center, 9849 Kenworthy St, EI Paso, TX 79924, USA, e-mail: justin.wright@ ttuhsc.edu

\section{INTRODUCTION}

As a reliable, safe and cost effective medical imaging modality, ultrasound is no longer limited to radiology, but is rapidly expanding into other specialties such as obstetrics and gynecology, anesthesiology, emergency medicine, family medicine, critical care, internal medicine, pediatrics, orthopedics and surgery. The hand-held ultrasound scanner was born out from advances in technology including miniaturization, image quality, portability, data storage and transmission, and longer battery- time. In recent years, the US Food and Drug Administration (FDA) has approved many new generation portable ultrasound devices including the Acuson P10 (Siemens) (Fig. 1), Vscan (GE) (Fig. 2), Signos RT Personal Ultrasound (Signostics) (Fig. 3) or MobiUS (Mobisante) (Fig. 4). As a result, hand-held ultrasound scanner becomes increasingly available in many clinical settings such as point of care, telemedicine and/ or rural medicine. Potential users of hand-held ultrasound raise many questions concerning the image quality of these new instruments in comparison to those of the standard ultrasound units. In addition, because ultrasound imaging is highly operator-dependent, given the variety of providers that may be using ultrasound in point-of-care, telemedicine and rural medicine, standards must be established for the level of training and competency in the operation and interpretation of hand-held ultrasound.

\section{Advantages and Disadvantages of Hand-Held Ultrasound Scanners}

Stethoscope, the symbol of modern medicine, has been in place for more than 150 years. However, this reliable diagnostic instrument has started to show its age in the era of evidence-based medicine when it's compared with a newer diagnostic instrument: the hand-held ultrasound.

Several studies demonstrated that medical students and residents can improve the accuracy of their physical examinations with the use of hand-carried ultrasound. ${ }^{1-3}$ Similarly, physical examination performed by hospitalists with hand-carried ultrasound are far more accurate than those performed without the instrument in the diagnosis of left ventricular function, cardiomegaly and pericardial effusion, but not for the assessment of valvular heart disease. ${ }^{4}$ However, the authors did not provide evidences of clinical benefits owing to more accurate physical examination. 


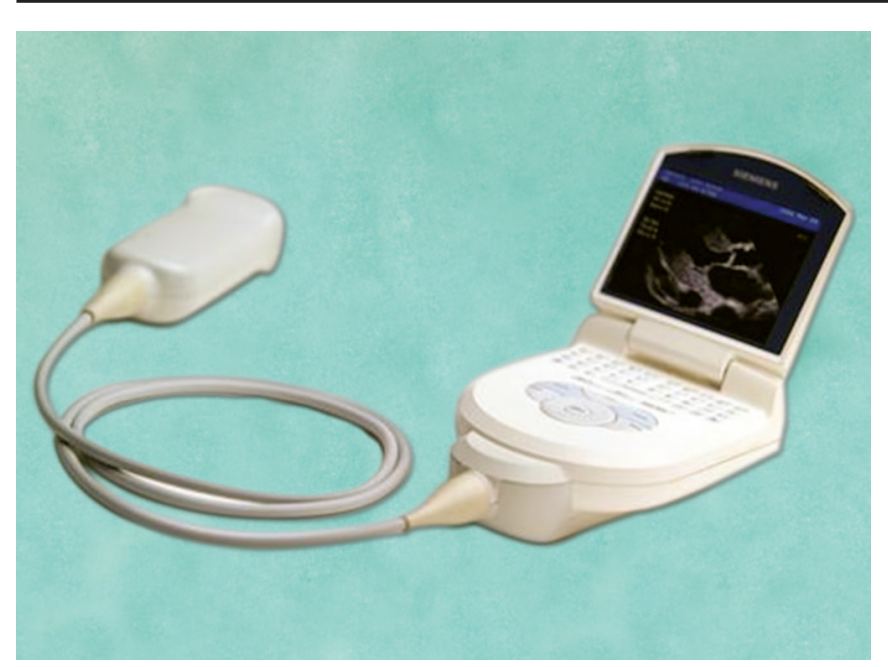

Fig. 1: Hand-held ultrasound Acuson P-10, Siemens

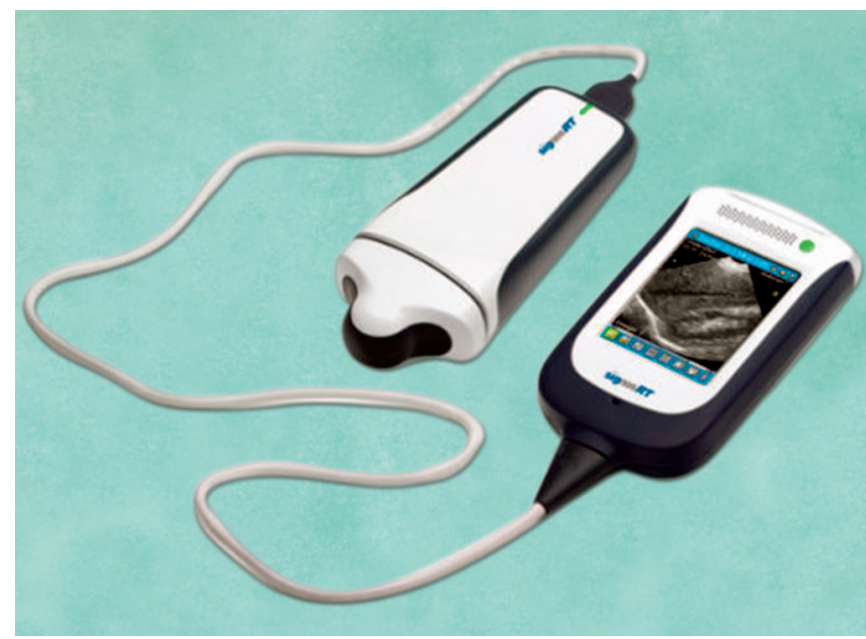

Fig. 3: Hand-held ultrasound Signos RT, Signostics

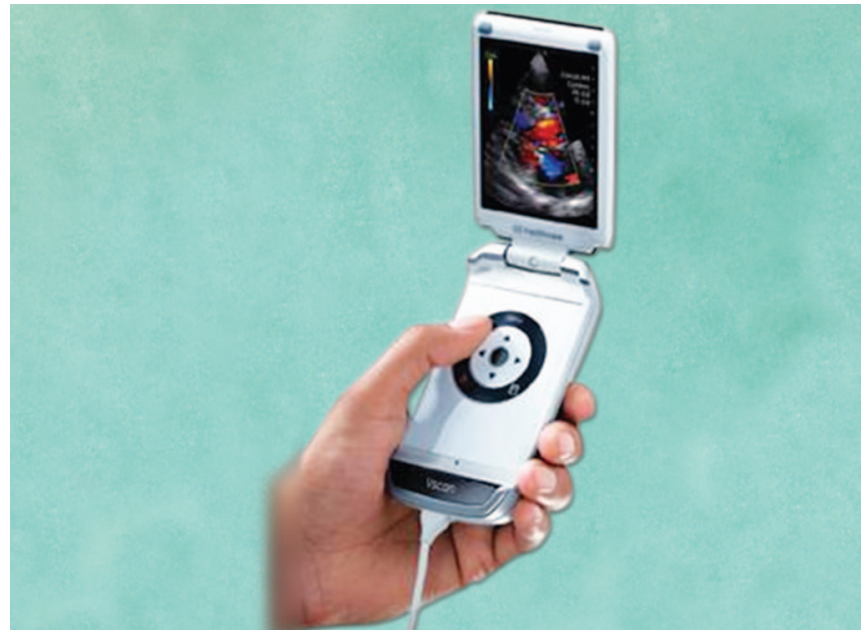

Fig. 2: Hand-held ultrasound Vscan, General Electric

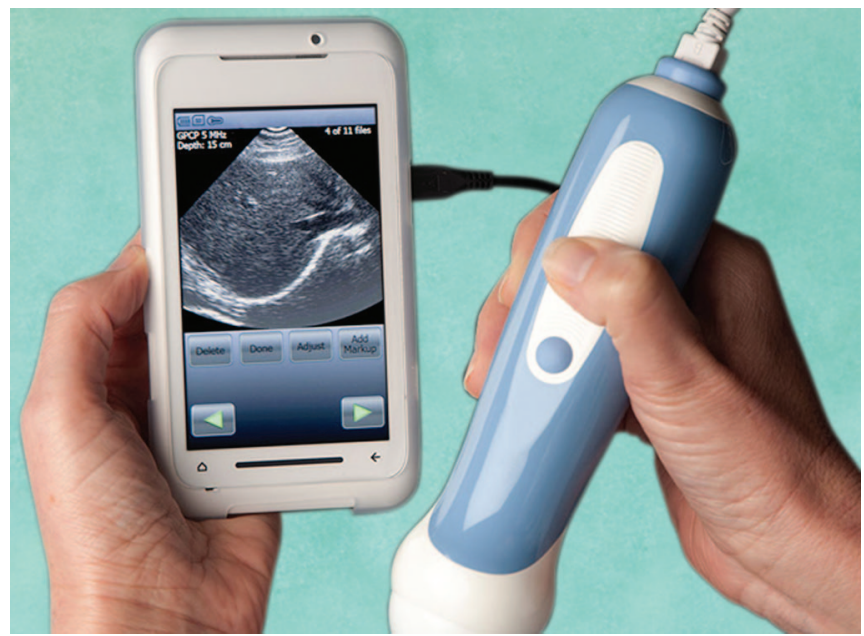

Fig. 4: Hand-held ultrasound MobiUS TC1, Mobisante

Table 1: Advantages and disadvantages of hand-held ultrasound

\begin{tabular}{|c|c|c|}
\hline Characteristics & Advantages & Disadvantages \\
\hline Portability & $\begin{array}{ll}\text { - } & \text { Availability for bedside, } \mathrm{POC} \text { or rural } \\
& \text { settings } \\
\text { - } & \text { Ease of use } \\
\text { - } & \text { Light weight } \\
\text { - } & \text { Short setup time }\end{array}$ & $\begin{array}{ll} & \text { Less features than standard US } \\
\text { - } & \text { Issues with Medicare reimbursement }\end{array}$ \\
\hline Low cost & $\begin{array}{l}\text { - Availability for bedside, POC or rural } \\
\text { setting } \\
\text { - Easy accessibility }\end{array}$ & - Less features than standard US \\
\hline Battery-operated & $\begin{array}{l}\text { Availability on fields or in remote } \\
\text { settings }\end{array}$ & $\begin{array}{l}\text { - Limited operation time (about 1-hour } \\
\text { of continuous operation) }\end{array}$ \\
\hline Transducer & - Integrated (Vscan) & - Limited choices \\
\hline Image quality & - Good quality with trained operator & - Inferior to standard US \\
\hline Storage capacity & - Removable media & - Limited capacity \\
\hline $\begin{array}{l}\text { Accuracy in the diagnosis } \\
\text { of diseases }\end{array}$ & $\begin{array}{l}\text { Improve accuracy of bedside physical } \\
\text { examination }\end{array}$ & $\begin{array}{l}\text { - Inferior to standard US } \\
\text { - } \quad \text { Higher rates of false-positive and } \\
\text { false-negative diagnoses }\end{array}$ \\
\hline Recording format & $\begin{array}{ll}\text { - } & \text { DICOM } \\
\text { - } & \text { JPEG } \\
\text { - } & \text { Wireless transmission (MobiUS) }\end{array}$ & - Issue for integration into EMR \\
\hline
\end{tabular}

POC: point of care; EMR: electronic medical record 
There are many advantages in using hand-held ultrasound, as demonstrated by numerous clinical studies investigating the usability, accuracy, and quality of these devices in different clinical settings and specialties. ${ }^{4-7}$ However, many disadvantages of hand-held ultrasound were also reported in these studies ${ }^{8-10}$ (Table 1).

\section{Benefits of Hand-Held Ultrasound in Obstetrics and Gynecology Practice}

The availability of ultrasonography in the management of patients at bedside, point-of-care and rural areas has changed the role of this excellent diagnostic tool in the practice of medicine. The safety and portability of hand-held ultrasound devices has allowed many medical specialties to adopt the new instruments to enhance the accuracy of physical examination and improve clinical performance.

The United Nations Children's Fund (UNICEF) estimated that over 500,000 women from developing countries die every year owing to complications of pregnancy and childbirth. Countries from sub-Saharan Africa and South Asia account for $84 \%$ of the maternal deaths. Poverty, inequity, cultural and/or traditional practices may prevent women from accessing delivery or postpartum care. ${ }^{11}$

There is a paucity of literature in investigating the application of hand-held ultrasound in obstetrics and gynecology. However, some authors had demonstrated the value of these devices in aiding the diagnosis of different diseases including trauma-related conditions, intraperitoneal bleeding, ectopic pregnancy, fetal evaluation and gynecological pathologies such as assessment of abnormal endometrial thickening, ovarian and uterine masses. ${ }^{4,5,12,13}$

Luque et al made a transvaginal probe with a simple adapter snapped over the Vscan transducer cable. ${ }^{12}$ The author compared ultrasound image quality of 80 patients (25 obstetric and 55 gynecological patients) examined by the Vscan and the ultrasound standard equipment, Voluson 730 -E. The Vscan was able to detect $98.75 \%$ of all lesions detected by the V730-E. In addition, to assess the Vscan capability in telemedicine, 20 still images with and without annotation, were sent by email to a remote station. There were no degradation of the image quality and the average time for transmitting image was 40 seconds.

Zalud compared the performance of the hand-held ultrasound Acuson P-10 with that of the formal ultrasound instrument in evaluating 200 patients referred for standard obstetrics and gynecology ultrasound exams such as amniotic fluid measurement, determination of fetal presentation, position of the placenta, detection of fetal heart activity and fetal movements and evaluation of the uterus and adnexal lesions. ${ }^{13}$ The study showed that hand-held ultrasound was reliable when making the initial diagnosis required by the limited ultrasound exam in obstetrics and gynecology but necessary caution must be taken when examining patients with placenta previa and intrauterine growth restriction (IUGR). ${ }^{13}$

\section{Does Hand-Held Ultrasound Offer Faster, But Not Necessarily Better Diagnosis?}

As the availability of portable ultrasound devices continue to increase and with these machines getting even smaller, the use of hand-held units confronts many challenges. They are more lightweight, durable and affordable, as well as relatively easy to use. Some hand-held machines have inbuilt power unit.

Another study examined the potential usefulness of hand-held ultrasound examination in the diagnosis of pleural, peritoneal or pericardial effusion and deep vein thrombosis in 169 patients. Patients were followed up to determine the presence or absence of the lesions. Median duration of examination was 6 minutes. The suspected lesion was found in 45 cases (17\%). Ultrasonographic examination improved diagnostic accuracy in $181(67 \%)$ cases. $^{5}$

Blaivas et al found lower quality in resolution and total image quality of the hand-held ultrasound Sonosite 180 Plus when they compared it with a standard ultrasound machine, GE $400 .{ }^{9}$ On contrary, other authors demonstrated comparable detection capability of GE Vscan and standard equipment, the Voluson 730 Expert. ${ }^{12}$ However, all the authors agreed that the hand-held ultrasound requires much less time for setup.

\section{Does the use of Hand-Held Ultrasound Increase the Likelihood of False Positive/Negative Diagnoses?}

A pilot study was carried out to demonstrate the clinical impact of hand-carried ultrasound in the management of 72 patients at a medical clinic. ${ }^{14}$ In this study, 9 residents without previous echocardiography experience participated in the study after completing a 20-hour training program on focused echocardiography. After the training period, residents were able to acquire images of diagnostic quality in $94 \%$ of patients. The ultrasound findings helped changing the medical management in over $40 \%$ of the patients. ${ }^{14}$ Despite these favorable results, however, the overuse and misuse of hand-held ultrasound, together with the lack of formal training and standard measures for clinical competency, and the limited features of hand-held ultrasound devices can result in inaccuracy of the diagnosis. As a result, falsenegatives may lead to a potential harm to the patients, while false-positive findings may subject patients to unnecessary invasive diagnostic or therapeutic procedures. These 
diagnostic errors, fortunately, were rare in reported studies on the use of hand-held ultrasound. ${ }^{12,14,15}$

\section{Minimum Standards for Training, Competence and Quality Assurance in Hand-Held Ultrasound}

Ultrasound imaging is highly operator-dependent, with the quality and utility of images reliant upon the training and skill of the provider performing the test. Given the variety of providers that may be using ultrasound in a rural setting, the level of understanding and scope of ultrasound use is dependent upon which personnel is available in the setting.

Physicians who have completed a residency program in obstetrics and gynecology receive extensive training in diagnostic ultrasound. ${ }^{16}$ The American Institute for Ultrasound in Medicine (AIUM) guidelines state that physicians performing diagnostic ultrasound examinations in their field (in this case, prenatal ultrasounds) should complete an approved residency program, fellowship, or postgraduate training that includes at least 3 months of dedicated diagnostic ultrasound education and have evidence of performing, evaluating, and interpreting at least 300 sonograms. ${ }^{16,17}$ Graduates of select family medicine residency programs are also taught to perform prenatal ultrasound through a series of didactic lectures and supervised scanning, showing competence in 25 to 50 scans. ${ }^{18}$ In order to comply with the AIUM guidelines, those physicians should show documentation of 100 AMA PRA Category 1 Credits ${ }^{\mathrm{TM}}$ dedicated to diagnostic ultrasound and evidence of performing, evaluating and interpreting the images of at least 300 sonograms within a 3 -year period. ${ }^{17}$

By virtue of their ultrasound training, these providers should be capable of a full ultrasound examination, determining fetal well-being, fetal position, placental position, as well as determining any fetal anomalies. They should also be able to identify any urgent or emergent issues that may arise, often without the aid of a central expert provider, utilizing telemedicine for consultation only. But what about the provider with little ultrasound training who may encounter urgent or emergent issues?

There have been several programs instituted to train providers (physician and nonphysician) in more limited and focused ultrasound examinations. Trainees in an emergency medicine residency were able to determine the presence or absence of an intrauterine pregnancy (IUP) in patients for whom ectopic pregnancy was suspected after a 1-hour didactic lecture, 10-question competency exam and minimum of 10 supervised transvaginal ultrasounds. ${ }^{19}$ Obstetric nurses have been trained to perform Limited Obstetric Ultrasonography (LOBU) to assess fetal wellbeing, fetal presentation, fetal number and placenta location.
This training was achieved with 12 hours of didactic education and clinical practicum consisting of 6 to 9 hours and approximately 15 scans. ${ }^{20}$ A nonphysician astronaut was trained to perform a focused assessment with sonography for trauma (FAST) examination after a 3-hour training covering hardware familiarization and ultrasound basics. ${ }^{21}$ He also had access to a reference card with layout coding for equipment controls, topologic points of reference to facilitate probe placements and probe manipulation terminology to aid in scanning. He was guided by an ultrasound imaging expert at mission control, transmitting images to earth from the International Space Station.

\section{Strategy to Train the Users of Hand-Held Ultrasound}

The experiences of the emergency medicine residents, obstetric nurses and the International Space Station demonstrate that it is possible to teach limited ultrasound examinations using a short-term combined didactic and supervised scanning curriculum. This strategy may be used to train providers to use hand-held ultrasound in emergency situations under the guidance of a centralized expert in a telemedicine setting. Didactic sessions may be used to introduce the providers to the physics of ultrasound, machine usage, probe manipulation and key anatomic structures. Supervised hands-on sessions are used to enhance technical skills. The duration and number of these courses will vary, depending on the skills required (fetal presentation, placental location, identification of IUP, etc.). The courses may be repeated on an annual or biannual basis to maintain and/or to learn new skills.

\section{Who is in-charge of Monitoring the Quality of Hand-Held Ultrasound Exams?}

The utility of ultrasound imaging relies on quality images obtained by the scanner. A review of quality control measures in obstetric ultrasound discussed two approaches to quality control in obstetric ultrasound: quantitative and qualitative. ${ }^{22}$ Quantitative quality control is based on the analysis of a distribution of measurements compared to normative distributions, such as with nuchal translucency or biometric measurements. Qualitative quality control involves scoring images based on comparison with standard images required for a given study. ${ }^{22}$ Qualitative quality control may be the most useful in a rural setting, as the volume of scans done by any one provider may not provide enough of a distribution to be compared to normative distributions. Real-time feedback may also provide quality control. As shown in the use of ultrasound at the International Space Station, the provider was guided in real-time by the Earth-based expert, which 
provided immediate feedback and ensured the high quality of images. This same feedback can be used between a rural provider and the central expert to ensure an adequate and quality study.

\section{CONCLUSION}

The arrival of hand-held ultrasound devices has transformed point-of-care ultrasound into an emerging diagnostic tool that can potentially enhance the quality of physical examination and clinical decisions in many settings including telemedicine, rural medicine and particularly, maternal care facilities in developing countries. However, more clinical studies need to be done to demonstrate the impact of handheld ultrasound on the quality of care and patient outcome, especially in obstetrics and gynecology. In addition, it's of paramount importance for the establishment of standards in the training curriculum, measuring competency and defining quality of the hand-held ultrasound examinations to ensure beneficial effects on the patients.

\section{REFERENCES}

1. Decara JM, Kirkpatrick JN, Spencer KT, et al. Use of handcarried ultrasound devices to augment the accuracy of medical student bedside cardiac diagnoses. Journal of the American Society of Echocardiography: official publication of the American Society of Echocardiography. 2005 Mar;18(3): 257-263.

2. Afonso N, Amponsah D, Yang J, et al. Adding new tools to the black bag-introduction of ultrasound into the physical diagnosis course. Journal of General Internal Medicine 2010 Nov;25(11):1248-1252.

3. Kimura BJ, Amundson SA, Willis CL, Gilpin EA, DeMaria AN. Usefulness of a hand-held ultrasound device for bedside examination of left ventricular function. The American Journal of Cardiology 2002 Nov 1;90(9):1038-1039.

4. Martin LD, Howell EE, Ziegelstein RC, et al. Hand-carried ultrasound performed by hospitalists: does it improve the cardiac physical examination? The American Journal of Medicine 2009 Jan;122(1):35-41

5. Lapostolle F, Petrovic T, Lenoir G, et al. Usefulness of handheld ultrasound devices in out-of-hospital diagnosis performed by emergency physicians. The American Journal of Emergency Medicine 2006 Mar;24(2):237-242.

6. Vourvouri EC, Koroleva LY, Ten Cate FJ, et al. Clinical utility and cost effectiveness of a personal ultrasound imager for cardiac evaluation during consultation rounds in patients with suspected cardiac disease. Heart $2003 \mathrm{Jul} ; 89(7): 727-730$.

7. Vourvouri EC, Poldermans D, Deckers JW, Parharidis GE, Roelandt JR. Evaluation of a hand carried cardiac ultrasound device in an outpatient cardiology clinic. Heart 2005 Feb;91(2):171-176.
8. Kimura BJ, Amundson SA, Shaw DJ. Hospitalist use of handcarried ultrasound: preparing for battle. Journal of Hospital Medicine: an official publication of the Society of Hospital Medicine 2010 Mar;5(3):163-167.

9. Blaivas M, Brannam L, Theodoro D. Ultrasound image quality comparison between an inexpensive handheld emergency department (ED) ultrasound machine and a large mobile ED ultrasound system. Academic Emergency Medicine: official Journal of the Society for Academic Emergency Medicine 2004 Jul;11(7):778-781.

10. Blaivas M, Theodoro D. Intraperitoneal blood missed on a FAST examination using portable ultrasound. The American Journal of Emergency Medicine 2002 Mar;20(2):105-107.

11. UNICEF. Progress for Children: A Report Card on Maternal Mortality. September 2008, Number 7. Available at: http://www. unicef.org/childsurvival/files/Progress_for_Children-No._7_LoRes_082008.pdf.

12. Troyano Luque JM, Ferrer-Roca O, Barco-Marcellan MJ, Sabatel Lopez R, Perez-Medina T, Perez-Lopez FR. Modification of the hand-held Vscan ultrasound and verification of its performance for transvaginal applications. Ultrasonics 2013 Jan;53(1):17-22.

13. Zalud I. Ultrasound Frontiers: 3D Doppler, Automatic Fetal Biometry, Handheld Ultrasound Donald School Journal of Ultrasound in Obstetrics and Gynecology 2010;4(1):89-96.

14. Croft LB, Duvall WL, Goldman ME. A pilot study of the clinical impact of hand-carried cardiac ultrasound in the medical clinic. Echocardiography 2006 Jul;23(6):439-446.

15. Duvall WL, Croft LB, Goldman ME. Can hand-carried ultrasound devices be extended for use by the noncardiology medical community? Echocardiography 2003 Jul;20(5): 471-476.

16. Hall R, Ogburn T, Rogers RG. Teaching and evaluating ultrasound skill attainment: competency-based resident ultrasound training for AIUM accreditation. Obstet Gynecol Clin North Am United States 2006;33:305-323, ix.

17. AIUM. Training Guidelines for Physicians Who Evaluate and Interpret Diagnostic Ultrasound Examinations. 2011. Available at: http://www.aium.org/resources/viewStatement.aspx?id=14. Accessed April 8, 2013.

18. Dresang LT, Rodney WM, Dees J. Teaching prenatal ultrasound to family medicine residents. Family Medicine 2004 Feb;36(2): 98-107.

19. MacVane CZ, Irish CB, Strout TD, Owens WB. Implementation of transvaginal ultrasound in an emergency department residency program: an analysis of resident interpretation. J Emerg Med United States: Elsevier Inc 2012;43:124-128.

20. Stringer M, Miesnik SR, Brown LP, Menei L, Macones GA. Limited obstetric ultrasound examinations: competency and cost. Journal of Obstetric, Gynecologic, and Neonatal Nursing : JOGNN/NAACOG 2003 May-Jun;32(3):307-312.

21. Sargsyan AE, Hamilton DR, Jones JA, et al. FAST at MACH 20: clinical ultrasound aboard the International Space Station. J Trauma United States 2005;58:35-39.

22. Salomon LJ, Ville Y. The science and art of quality in obstetric ultrasound. Current Opinion in Obstetrics and Gynecology 2009 Apr;21(2):153-160. 